\title{
BOUNDARY VALUES OF ANALYTIC FUNCTIONS WITHOUT DISTRIBUTIONAL POINT VALUES
}

\author{
RICARDO ESTRADA
}

\begin{abstract}
We give a method to construct distributions that are boundary values of analytic functions which have non-tangential limits at points where the distributional point value does not exist.
\end{abstract}

\section{Introduction.}

Let $f \in \mathcal{D}^{\prime}(\mathbb{R})$ be a distribution that is the boundary value of an analytic function defined in the upper-half plane, $f(x)=F(x+i 0)$, distributionally. Then it is well-known that if the distributional point value $f\left(x_{0}\right)=L$ exists in the sense of Lojasiewicz [7], then $F\left(x_{0}+i y\right) \rightarrow L$ as $y \rightarrow 0^{+}[10,11]$.

The purpose of this note is to show how one can construct a counterexample to the reciprocal result. Namely, we will show that there are functions $F$, analytic in the upperhalf plane, with distributional boundary values $f(x)=F(x+i 0), f \in \mathcal{D}^{\prime}(\mathbb{R})$, for which

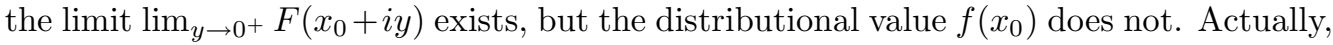
counterexamples where even the non-tangential limit of $F(z)$ as $z \rightarrow x_{0}$ exists can be constructed.

Our construction is based on the Baire theorem, and so we start by giving a useful variant of this result in Section 2. Next, in Section 3 we show the existence of series $\sum_{n=0}^{\infty} a_{n}$ that are Abel but not Cesàro summable and satisfy the additional condition $a_{n}=O\left(n^{\beta}\right), n \rightarrow \infty$, for some $\beta>-1$. The existence of such series is used in Section 4 to prove the existence of the announced counterexamples.

\section{A variant of the Baire Theorem}

Our construction is based on the well-known Baire theorem [5]. The theorem of Baire says that a complete metric space is of the second category. Sets of the second category are those that are not of the first category, i.e., countable unions of nowhere dense sets. A nowhere dense set is one whose closure has empty interior.

Received May 29, 2002.

2000 Mathematics Subject Classification. Primary 30B30, 46F12.

Key words and phrases. Boundary values of analytic functions, distributional point values. 
Our argument uses a variant of the Baire theorem, that although really useful, does not seem to be stated explicitly in the texts; thus, it is convenient to start the article by considering this result.

If $E$ is a closed proper linear subspace of a topological vector space $F$, then it is nowhere dense, since the only linear subspace with non-empty interior is $F$ itself. Thus is $\left\{E_{n}\right\}_{n=1}^{\infty}$ is a sequence of proper closed linear subspaces of a Fréchet space $F$, then $\bigcup_{n=1}^{\infty} E_{n} \neq F$. In the present situation we need to conclude that $\bigcup_{n=1}^{\infty} E_{n} \neq F$, where the $E_{n}$ 's are proper linear subspaces of $F$, but not closed (in fact, each of them is dense in $F$ !).

That $\bigcup_{n=1}^{\infty} E_{n}$ may be equal to $F$, where $F$ is any infinite dimensional Fréchet space, can be seen from the following construction. Let $\left\{x_{a}\right\}_{a \in A}$ be an algebraic (or Hamel) basis of $F$ over the field ( $\mathbb{R}$ or $\mathbb{C}$ ). Since $A$ is infinite, there is an increasing sequence of sets $\left\{A_{n}\right\}_{n=1}^{\infty}$ whose union is $A, A_{n} \nearrow A$. Let $E_{n}$ be the linear subspace spanned (algebraically) by $\left\{x_{a}\right\}_{a \in A_{n}}$. Then the $E_{n}$ are proper linear subspaces of $F$ and $\bigcup_{n=1}^{\infty} E_{n}=F$. As we show in the Theorem 2 , however, the conclusion $\bigcup_{n=1}^{\infty} E_{n} \neq F$ can be obtained under additional hypothesis on the $E_{n}$ 's.

Theorem 1. Let $E$ and $F$ be Fréchet spaces. Suppose $E \subset F$ and the inclusion is continuous. If $E \neq F$ then $E$ is of the first category in $F$.

Proof. Denote as $\mathfrak{T}_{E}$ and $\mathfrak{T}_{F \rightarrow E}$ the topologies of $E$ as a Fréchet space and as a subspace of $F$, respectively. We are assuming that the identity map $I d:\left(E, \mathfrak{T}_{E}\right) \rightarrow$ $\left(E, \mathfrak{T}_{F \rightarrow E}\right)$ is continuous.

If $I d$ is also open, then the two topologies coincide. It then follows that $\left(E, \mathfrak{T}_{F \rightarrow E}\right)$ is complete and, consequently, that $E$ is a closed subspace of $F$. Since $E \neq F$ it follows that $E$ is nowhere dense in $F$ and thus of the first category.

Let us now consider the situation when $I d$ is not open. Let $d_{E}$ and $d_{F}$ be invariant metrics for $E$ and $F$, respectively. Consider the "balls"

$$
B_{E, r}(0)=\left\{x \in E: d_{E}(x, 0) \leq r\right\} .
$$

Let $W_{r}$ be the closure of $B_{E, r}(0)$ in $F$. Then either (i) $W_{r}$ is a neighborhood of zero in $F$ for each $r>0$, or (ii) there exists $r_{0}>0$ such that the origin is not in the interior of $W_{r}$ for $r \leq r_{0}$.

If (ii) holds, then the interior of $W_{r}$ is empty if $r \leq r_{0} / 2$, since if $x \in \operatorname{int}\left(W_{r}\right)$ then $0 \in \operatorname{int}\left(W_{2 r}\right)$. Therefore $B_{E, r}(0)$ is nowhere dense in $F$ for $r \leq r_{0} / 2$ and consequently $E=\bigcup_{n=1}^{\infty} n B_{E, r}(0)$ is of the first category in $F$.

Let us show that (i) cannot hold, since it would imply that $E=F$. Indeed, when (i) holds, then $\forall r>0 \exists \rho=\rho(r)>0$ such that $B_{F, \rho}(0) \subset W_{r}$. Fix $r=r_{0}$ and $\rho=\rho(r)$. Let $y \in B_{F, \rho}(0)$. Let $r_{n} \searrow 0$ be a decreasing sequence such that $\sum_{n=1}^{\infty} r_{n}<\infty$, and let $\rho_{n}=\rho\left(r_{n}\right)$, chosen in such a way that $\rho_{n} \searrow 0$. Then a sequence $\left\{x_{n}\right\}_{n=1}^{\infty}$ of $E$ can be constructed recursively by requiring that

$$
d_{F}\left(y, \sum_{j=1}^{n} x_{j}\right)<\rho_{n},
$$




$$
d_{E}\left(x_{n}, 0\right)<r_{n-1} .
$$

Then (2.3) implies that the partial sums of the series $\sum_{n=1}^{\infty} x_{n}$ form a Cauchy sequence in $E$ and, since $E$ is complete, $\sum_{n=1}^{\infty} x_{n}$ converges in $E$ to some $x \in E$. But this yields that $\sum_{n=1}^{\infty} x_{n}=x$ in the topology of $F$ too, while (2.2) gives that $\sum_{n=1}^{\infty} x_{n}=y$ in $F$. We conclude that $y=x \in E$. Thus $B_{F, \rho}(0) \subset E$ and so $F=\bigcup_{n=1}^{\infty} n B_{F, \rho}(0) \subset E$.

Using the Theorem 2.1 we immediately obtain our variant of the Baire theorem.

Theorem 2. Let $F$ be a Fréchet space. Let $\left\{E_{n}\right\}_{n=1}^{\infty}$ be a sequence of Fréchet spaces such that $E_{n} \subset F$, inclusions being continuous. If $E_{n} \neq F \forall n$ then $\bigcup_{n=1}^{\infty} E_{n} \neq F$.

It is worth remarking that local convexity is not used in these results, so that they hold for complete metrizable topological vector spaces, not just Fréchet spaces.

\section{Series that are Abel but not Cesàro Summable}

Let $\sum_{n=0}^{\infty} a_{n}$ be a possibly divergent series. We say that the series is Abel summable to $S$, and write

$$
\sum_{n=0}^{\infty} a_{n}=S \quad(\mathrm{~A}),
$$

if the series $\sum_{n=0}^{\infty} a_{n} r^{n}$ converges for $0 \leq r<1$ and

$$
\lim _{r \rightarrow 1} \sum_{n=0}^{\infty} a_{n} r^{n}=S .
$$

Notice that to each Abel summable series there corresponds an analytic function $F(z)=$ $\sum_{n=0}^{\infty} a_{n} z^{n}$ which is defined in the unit disc $\Delta=\{z \in \mathbb{C}:|z|<1\}$ and that has a radial limit at $z=1$, and conversely. The space of Abel summable series $F_{\text {Abel }}$ has a natural structure of a Fréchet space, corresponding to the intersection topology of the Fréchet space $H(\Delta)$ of analytic functions in $\Delta[9]$, and the Banach space $C[0,1]$, with its usual supremum norm.

On the other hand, the Cesàro summability of the series $\sum_{n=0}^{\infty} a_{n}$ is defined as follows $[3,4]$. Set

$$
\begin{gathered}
A_{n}^{0}=a_{0}+\cdots+a_{n}, \\
A_{n}^{\kappa}=A_{0}^{\kappa-1}+\cdots+A_{n}^{\kappa-1} .
\end{gathered}
$$

Then the series is $(\mathrm{C}, \kappa)$ summable to $S$, written as

$$
\sum_{n=0}^{\infty} a_{n}=S \quad(\mathrm{C}, \kappa),
$$

if

$$
\lim _{n \rightarrow \infty} \frac{A_{n}^{\kappa}}{n^{\kappa}}=\frac{s}{\kappa !} .
$$


The notation $\sum_{n=0}^{\infty} a_{n}=S$ (C) means that (3.5) holds for some $\kappa$. The formula

$$
A_{n}^{\kappa}=\sum_{q=0}^{n}\left(\begin{array}{c}
q+\kappa \\
\kappa
\end{array}\right) a_{n-q},
$$

that makes sense for any $\kappa \in \mathbb{R}, \kappa>-1$, allows us to define the Cesàro summability of non-integral order. Since when $\kappa_{1}>\kappa_{2}$, then $\left(\mathrm{C}, \kappa_{1}\right)$ summability implies $\left(\mathrm{C}, \kappa_{2}\right)$ summability, for our present purposes one may consider only summability of intergral order.

Convergence implies $(\mathrm{C}, \kappa)$ summability of any order, while Cesàro summability, in turn, implies Abel summability.

It is very simple to construct series that are Abel but not Cesàro summable. Indeed, if $\sum_{n=0}^{\infty} a_{n}$ is the series obtained by setting $x=1$ in the Taylor series $\sum_{n=0}^{\infty} a_{n} x^{n}=$ $e^{(1+x)^{-1}}$, then $\sum_{n=0}^{\infty} a_{n}=e^{1 / 2}(\mathrm{~A})$, but the series is not $(\mathrm{C})$ summable since the terms are not of the order $O\left(n^{\beta}\right)$ for any $\beta \in \mathbb{R}$. Similarly, the series $\sum_{n=0}^{\infty}(-1)^{n} e^{c \sqrt{n}}$, where $c>0$, is also (A) but not (C) summable [3, 4].

The counterexample of Section 4, however, requires the construction of a series $\sum_{n=0}^{\infty} a_{n}$ that is Abel but not Cesàro summable and whose terms satisfy a bound of the type $a_{n}=O\left(n^{\beta}\right), n \rightarrow \infty$, for some $\beta \in \mathbb{R}$. That this is not possible if $\beta=-1$ follows from Littlewood's tauberian theorem [6], since if $\sum_{n=0}^{\infty} a_{n}=S(\mathrm{~A})$ and $a_{n}=O\left(n^{-1}\right)$, $n \rightarrow \infty$, then $\sum_{n=0}^{\infty} a_{n}$ actually converges to $S$. Nevertheless, such examples exist for any $\beta>-1$.

Theorem 3. Let $\beta>-1$. Then there exists a series $\sum_{n=0}^{\infty} a_{n}$ with $a_{n}=O\left(n^{\beta}\right), n \rightarrow$ $\infty$, that is Abel but not Cesàro summable.

Proof. Let $F_{\beta}$ be the Banach space of series $\sum a_{n}$ of complex terms that satisfy $a_{n}=O\left(n^{\beta}\right), n \rightarrow \infty$, with the norm

$$
\left\|\left\{a_{n}\right\}\right\|_{F_{\beta}}=\max \left\{\left|a_{0}\right|, \sup _{n \geq 1} n^{-\beta}\left|a_{n}\right|\right\} .
$$

Let $F_{\text {Abel }}$ be the Fréchet space of (A) summable series, introduced before, and let $F=$ $F_{\beta} \cap F_{\text {Abel }}$, with the intersection topology; interestingly, $F$ is not only a Fréchet space but actually a Banach space.

For any $\kappa>1$, let $E_{(\mathrm{C}, \kappa)}$ be the space of series that are $(\mathrm{C}, \kappa)$ summable, with the seminorms

$$
\left\|\left\{a_{n}\right\}\right\|_{E_{(\mathrm{C}, \kappa)}}=\sup _{n \geq 1} n^{-\kappa}\left|A_{n}^{\kappa}\right| \Gamma(\kappa+1),
$$

where the Cesàro means of order $\kappa$ are given by (3.7). Let $E_{\kappa}=E_{(\mathrm{C}, \kappa)} \cap F_{\beta}$ with the intersection topology. Since $E_{\kappa}$ is a is Banach space, and the inclusion $E_{\kappa} \rightarrow F$ is continuous, if we show that $E_{\kappa} \neq F \forall \kappa$, it will follow that $E_{\kappa}$ is of the first category in $F$ and, consequently, that $\bigcup_{\kappa>-1} E_{\kappa} \neq F$, which is precisely what we are required to show. 
But to show that $E_{\kappa} \neq F$ for any $\kappa$, since the $E_{\kappa}$ are increasing with $\kappa$, it is enough to show that $E_{\kappa_{1}} \neq E_{\kappa_{2}}$ if $\kappa_{1} \neq \kappa_{2}$. But [4, Section 6.12] the series

$$
\sum_{n=1}^{\infty} n^{b+i c} e^{A i n^{a}}
$$

where $0<a<1, b>-1$, is $(\mathrm{C}, \kappa)$ summable if and only if

$$
(\kappa+1) a-b>1 .
$$

If $\kappa_{1}<\kappa_{2}$, by choosing the parameters $a$ and $b, 0<a<1, b>-1$ in a way that

$$
b \leq \beta, \quad b<\kappa_{2}, \frac{b+1}{\kappa_{1}+1}>\alpha>\frac{b+1}{\kappa_{2}+1},
$$

we obtain that the series (3.10) belongs to $E_{\kappa_{1}} \backslash E_{\kappa_{2}}$.

Notice that the same procedure produces series that are $(\mathrm{A})$ but not $(\mathrm{C})$ summable and satisfy other extra conditions. For instance, we may ask $\left\{a_{n}\right\} \in l^{p}$ for $1<p<\infty$.

\section{Distributions without Point Values}

Let $f \in \mathcal{D}^{\prime}(\mathbb{R})$ be a distribution. Then $[3,7] f$ has a distributional point value in the sense of Eojasiewicz at $x=x_{0}$, written as

$$
f\left(x_{0}\right)=L \text { distributionally, }
$$

if

$$
\lim _{\varepsilon \rightarrow 0} f\left(x_{0}+\varepsilon x\right)=L,
$$

in the topology of $\mathcal{D}^{\prime}(\mathbb{R})$, that is, if

$$
\lim _{\varepsilon \rightarrow 0}\left\langle f\left(x_{0}+\varepsilon x\right), \phi(x)\right\rangle=L \int_{-\infty}^{\infty} \phi(x) d x, \quad \forall \phi \in \mathcal{D}^{\prime}(\mathbb{R}) .
$$

It can be shown that $f\left(x_{0}\right)=L$ distributionally if and only if there exist $N \in \mathbb{N}$ and a primitive of order $N$ of $f, F^{(N)}=f$, that is continuous in a neighborhood of $x=x_{0}$ and satisfies

$$
\lim _{x \rightarrow x_{0}} \frac{F(x)}{\left(x-x_{0}\right)^{N}}=\frac{L}{N !} .
$$

Let now $f \in \mathcal{D}^{\prime}(\mathbb{R})$ be a periodic distribution of period $2 \pi$, with Fourier series expansion

$$
f(\theta)=\sum_{n=-\infty}^{\infty} a_{n} e^{i n \theta}
$$


Then [1] $f\left(\theta_{0}\right)=L$ distributionally if and only if certain Cesàro averages of the Fourier series for $\theta=\theta_{0}$ converge to $L$, namely, if and only if

$$
\lim _{x \rightarrow \infty} \sum_{-x \leq n \leq a x} a_{n} e^{i n \theta}=L \quad(\mathrm{C}) \quad \forall a>0 .
$$

In particular $[1,10]$, if the Fourier series of $f$ is of the power series type, $f(\theta)=$ $\sum_{n=0}^{\infty} a_{n} e^{i n \theta}$, then

$$
f\left(\theta_{0}\right)=L \quad \text { distributionally } \Leftrightarrow \sum_{n=0}^{\infty} a_{n} e^{i n \theta_{0}}=L \quad(\mathrm{C}) .
$$

Let now $F(z)$ be an analytic function defined in the unit disc $\Delta=\{z \in \mathbb{C}:|z|<1\}$. Let

$$
F(z)=\sum_{n=0}^{\infty} a_{n} z^{n}
$$

be its Taylor series at $z=0$. Then $F$ has distributional boundary limits at $S=\{\xi \in \mathbb{C}$ : $|\xi|=1\}$, that is,

$$
f(\xi)=\lim _{r \rightarrow 1^{-}} F(r \xi),
$$

exists in $\mathcal{D}^{\prime}(S)$ if and only if one of the following two equivalent conditions is satisfied $[2]:$

1. $\exists M>0, \alpha \in \mathbb{R}$ such that $|F(z)| \leq M(1-|z|)^{-\alpha} \quad \forall z \in \Delta$;

2. $\exists \beta \in \mathbb{R}$ such that $a_{n}=O\left(n^{\beta}\right)$ as $n \rightarrow \infty$.

Combining these results with those of the previous section, we immediately obtain.

Theorem 4. There exists an analytic function defined in $|z|<1$, that has distributional boundary values at $|z|=1, f(\xi)=\lim _{r \rightarrow 1^{-}} F(r \xi)$, where $f \in \mathcal{D}^{\prime}(S)$, such that

(a) $\lim _{r \rightarrow 1^{-}} F(r)$ exists

(b) $f\left(e^{i \theta}\right)$ does not have a distributional value at $\theta=0$.

Proof. Let $\sum_{n=0}^{\infty} a_{n}$ be a series that is Abel but not Cesàro summable and that satisfies $a_{n}=O\left(n^{\beta}\right), n \rightarrow \infty$ for some $\beta \in \mathbb{R}$. Then $F(z)=\sum_{n=0}^{\infty} a_{n} z^{n}$ satisfies all the required conditions.

Observe that we may ask the boundary function $f$ to belong, for instance, to smaller spaces such as $H^{2}$. Notice also that we may construct a function $F$ as in Theorem 4 for which not only the radial limit but the non-tangential limit exists as $z=1$.

Using a conformal map we see that a corresponding result holds at any point of any smooth closed contour. In particular, there exists an analytic function $G(z)$ defined in the upper half-plane $\operatorname{Im} z>0$ that has distributional boundary values in $\mathbb{R}, g(x)=G(x+i 0)$, $g \in \mathcal{D}^{\prime}(\mathbb{R})$, such that 
(a) $\lim _{y \rightarrow 0} G\left(x_{0}+i y\right)$ exists (or more generally $\lim _{\substack{z \rightarrow x_{0} \\ \text { N.T. }}} G(z)$ exists.)

(b) $g(x)$ does not have a distributional point value at $x=x_{0}$.

It is interesting to observe that another application of the Baire theorem argument allows us to construct a function $f \in H^{2}$ such that the distributional point values $f(x)$ do not exists for $x \in X$, where $X$ is a dense $G_{\delta}$ subset of $S$. On the other hand, it is well-known [8] that if $f \in H^{2}$ is the boundary value of the analytic function $F(z)$ then $\lim _{r \rightarrow 1} F(r \xi)$ exists almost everywhere, that is, for all $\xi \in Y$ where $Y$ is a subset of full measure of $S$. However, this does not provide the counterexample of Theorem 4, since even though $X$ and $Y$ are very big subsets of $S$, in their own way, it could be that $X \cap Y=\emptyset$.

To construct such $f \in H^{2}$ and such dense $G_{\delta}$ set $X$ we proceed as follows. If $f \in H^{2}$, let $f(\theta)=\sum_{n=0}^{\infty} a_{n} e^{i n \theta}$ be its Fourier series expansion. Define

$$
\alpha_{\theta, k}(f)=\sup _{n \in \mathbb{N}}\left|A_{n}^{\kappa}(\theta)\right|
$$

where

$$
A_{n}^{\kappa}(\theta)=\sum_{q=0}^{n}\left(\begin{array}{c}
q+\kappa \\
q
\end{array}\right) a_{n-q} e^{i(n-q) \theta},
$$

are the $(\mathrm{C}, \kappa)$ means of the Fourier series at $\theta$. Fix $\theta$. Use of the series (3.10) shows that for each $\kappa$ there exists $f \in H^{2}$ with $\alpha_{\phi, \kappa}(f)=\infty$. Employing the Banach-Steinhaus theorem, we obtain that there exists a dense $G_{\delta}$ subset $B_{\theta}$ of $H^{2}$ such that $\alpha_{\theta, \kappa}(f)=\infty \forall \kappa$ if $f \in B_{\theta}$. Let $\left\{\theta_{n}\right\}_{n=1}^{\infty}$ be a dense countable subset of $[0,2 \pi]$ and let $B=\bigcap_{n=1}^{\infty} B_{\theta_{n}}$. Then $B$ is also a dense $G_{\delta}$ subset of $H^{2}$, and $\alpha_{\theta_{n}, \kappa}(f)=\infty \forall n, \kappa$ and $\forall f \in B$. But observe that for each fixed $f$ and $\kappa$ then $\alpha_{\theta, \kappa}(f)$ is an upper semicontinuous function of $\theta$. Therefore $X=\left\{\theta: \alpha_{\theta, \kappa}(f)=\infty\right.$ for some $\left.\kappa\right\}$ is a dense $G_{\delta}$ subset of $[0,2 \pi]$.

\section{References}

[1] Estrada, R., Characterization of the Fourier series of distributions having a value at a point, Proc. Amer. Math. Soc. 124(1996), 1205-1212.

[2] Estrada, R. and Kanwal, R. P., Distributional boundary values of analytic and harmonic functions, J. Math. Anal. Appl. 89(1982), 262-289.

[3] Estrada, R. and Kanwal, R. P., A Distributional Approach to Asymptotics. Theory and Applications, Second Edition, Birkhäuser, Boston, 2002.

[4] Hardy, G. H., Divergent Series, Clarendon Press, Oxford, 1949.

[5] Kelley, J. L. and Namioka, I., Linear Topological Spaces, Springer-Verlag, New York, 1976.

[6] Littlewood, J. E., The converse of Abel's theorem on power series, Proc. London. Math. Soc. 9(1911), 434-448.

[7] Łojasiewicz, S., Sur la valeur et la limite d'une distribution en un point, Studia Math. 16(1957), 1-36.

[8] Rudin, W., Real and Complex Analysis, McGraw Hill, New York, 1966.

[9] Treves, F., Topological Vector Spaces, Distributions and Kernels, Academic Press, New York, 1967. 
[10] Walter, G., Pointwise convergence of distribution expansions, Studia Math. 26(1966), 143154.

[11] Walter, G., Local behavior of harmonic functions, Illinois J. Math. 16(1970), 491-501.

Department of Mathematics, Louisiana State University, Baton Rouge, LA 70803, U.S.A.

E-mail: restrada@math.lsu.edu 